\title{
Very High Frequency Interleaved Self-Oscillating Resonant SEPIC Converter
}

\author{
Kovacevic, Milovan; Knott, Arnold; Andersen, Michael A. E.
}

Published in:

Proceedings of EPE '13-ECCE Europe

Publication date:

2013

Link back to DTU Orbit

Citation (APA):

Kovacevic, M., Knott, A., \& Andersen, M. A. E. (2013). Very High Frequency Interleaved Self-Oscillating Resonant SEPIC Converter. In Proceedings of EPE '13-ECCE Europe IEEE.

\section{General rights}

Copyright and moral rights for the publications made accessible in the public portal are retained by the authors and/or other copyright owners and it is a condition of accessing publications that users recognise and abide by the legal requirements associated with these rights.

- Users may download and print one copy of any publication from the public portal for the purpose of private study or research.

- You may not further distribute the material or use it for any profit-making activity or commercial gain

- You may freely distribute the URL identifying the publication in the public portal

If you believe that this document breaches copyright please contact us providing details, and we will remove access to the work immediately and investigate your claim 


\title{
Very High Frequency Interleaved Self-Oscillating Resonant SEPIC Converter
}

\author{
Milovan Kovacevic, Arnold Knott, Michael A. E. Andersen \\ Technical University of Denmark \\ Ørsteds Plads, Building 349 \\ 2800 Kgs. Lyngby, Denmark \\ Phone: +45 / 45255639 \\ Fax: +45 / 45880117 \\ Email: \{mikov, akn, ma\}@elektro.dtu.dk \\ URL: http://www.elektro.dtu.dk
}

\section{Keywords}

《very high frequency power converter», 《resonant converter》, 《zero voltage switching», 《interleaved converters》, 《self-oscillating》

\begin{abstract}
This paper describes analysis and design procedure of an interleaved, self-oscillating resonant SEPIC converter, suitable for operation at very high frequencies (VHF) ranging from $30 \mathrm{MHz}$ to $300 \mathrm{MHz}$. The presented circuit consists of two resonant SEPIC DC-DC converters, and a capacitive interconnection network between the switches which provides self-oscillating and interleaved operation. A design approach to ensure zero voltage switching (ZVS) condition of the MOSFET devices is provided.

To verify the proposed method, an $11 \mathrm{~W}, 50 \mathrm{MHz}$ prototype was built using low-cost VDMOS devices and experimental results are presented. Peak achieved efficiency was $87 \%$.
\end{abstract}

\section{Introduction}

A constant drive for miniaturization of power converters inevitably leads to increase of converter switching frequency. Reactive components scale down in both physical size and value, which permits use of power converters in applications with severe size constraints. This is especially beneficial regarding magnetic components, as they are typically the biggest contributors to the size of power converters. Inductance values required for operation at very high frequencies could easily be achieved with air core inductors, eliminating core loss entirely. For operation above $30 \mathrm{MHz}$, requirements for EMI filtering are significantly relaxed: EMI filters could be reduced to a few SMD components, or removed completely from a design. For some applications, for example LED drives, it is also beneficial if the power converter does not require electrolytic capacitors for voltage/current filtering, as they might limit the lifetime of LED lamps.

High switching frequencies impose new design challenges as well. With exception of very low power and voltage levels, hard-switched converters are unsuitable for operation at frequencies above a few $\mathrm{MHz}$ due to prohibitively large switching losses [1]. Generating signals for high-side switches is very difficult [2], which further limits topology selection, except in low-voltage converters [3]. Losses are further increased if hard gating is employed, which is exclusively the case for commercial DC-DC converters today. 


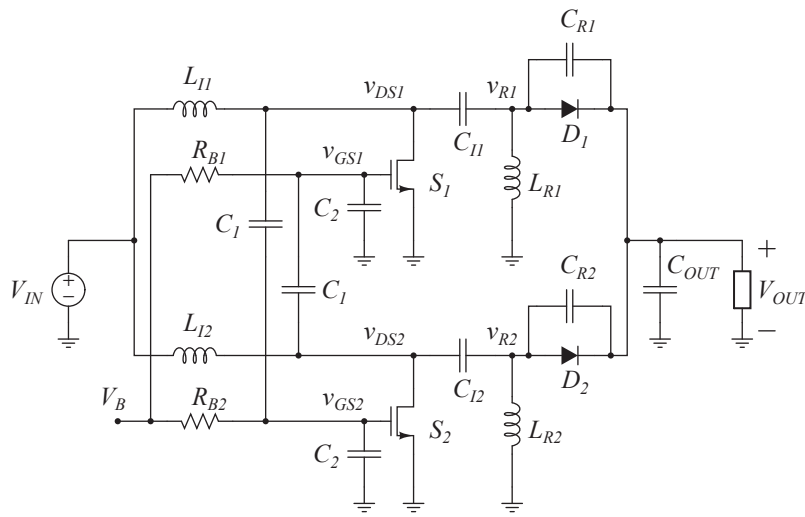

Fig. 1: Schematic of the interleaved, self-oscillating resonant SEPIC converter.

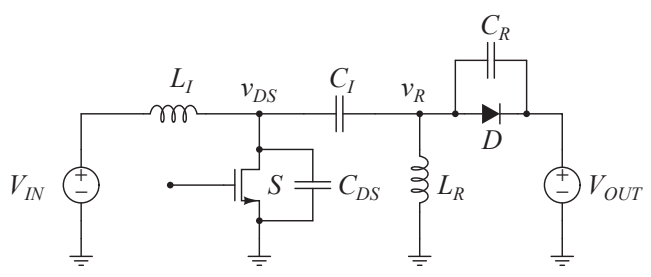

Fig. 2: Resonant SEPIC converter topology.
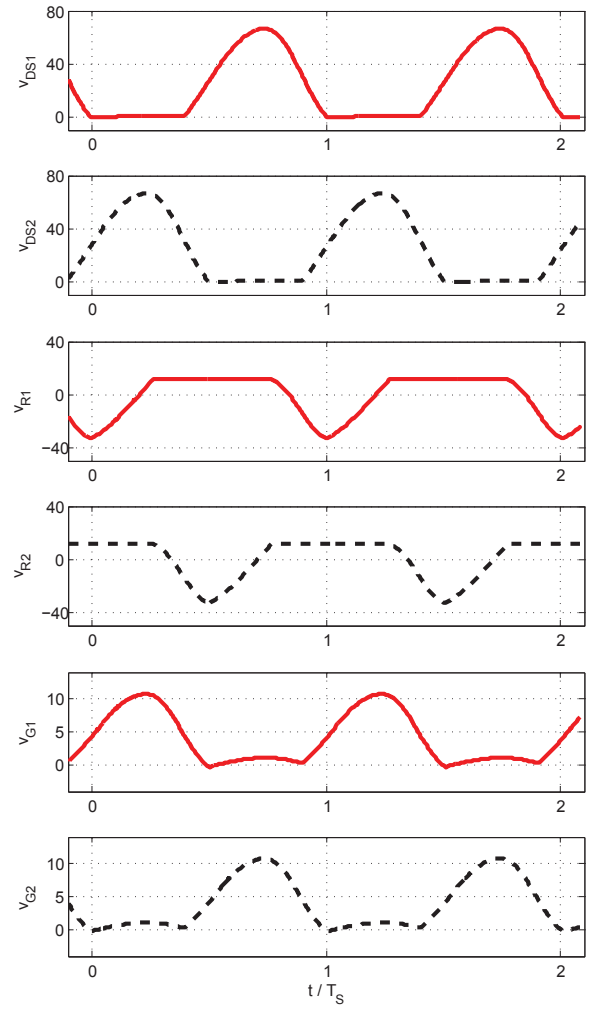

Fig. 3: Simulated waveforms of the drain, rectifier, and gate voltages of the interleaved self-oscillating resonant SEPIC converter; $V_{I N}=24 \mathrm{~V}$ and $V_{O U T}=12 \mathrm{~V}$.

To work around these problems, resonant converter topologies need to be considered in order to minimize or eliminate some of the switching loss mechanisms. Resonant [4-6] or multiresonant [1,7] gate driving techniques need to be employed as well, thereby reducing the gating loss by recovering portion of the energy from the gate capacitances. Since there are no gate driver solutions intended for operation above $30 \mathrm{MHz}$ currently available on the market, gate drivers for VHF converters are typically developed using discrete components.

This paper presents a recently developed technique to achieve interleaved, self-oscillating operation of two resonant converters, suitable for power conversion at frequencies in VHF (30 MHz - 300 MHz) range, and a design procedure for a step-down resonant SEPIC converter utilizing aforementioned technique. A general structure of the proposed converter, as well as the interconnection network which provides self-oscillating behavior and interleaved operation, are described. Design procedure for the proposed converter is provided. Finally, experimental results of an $11 \mathrm{~W}, 50 \mathrm{MHz}$ prototype are presented.

\section{Proposed Converter}

Fig. 1 shows the proposed converter topology. Two resonant SEPIC converters [7], as shown in Fig. 2, are coupled via capacitive interconnection network, which provides gate signal generation for driving the MOSFET devices. The circuit is symmetrical, and the gate voltages are in phase with the drain voltages of opposing MOSFETs due to capacitive voltage dividers; thus providing interleaved operation. Typical voltage waveforms of the interleaved resonant SEPIC converter are shown in Fig. 3. 
This section is organized as follows:

- topology and properties of the resonant SEPIC converter

- the interleaved converter topology

- the interconnection gate drive network

- converter startup

\section{Resonant SEPIC Converter}

Compared to the class E converter [4,8], which has bulky input and output inductances, in resonant SEPIC converter topology all reactive components are part of the resonant tank and therefore are small in value. Furthermore, fewer inductors are necessary, as well as lower component count in general [?]. This is particularly important if we have in mind that at RF frequencies inductors typically have low $Q$ factors due to skin and proximity effects, which increase conduction losses.

Due to presence of 5 resonant elements and 2 switching devices, even with ideal components the circuit in Fig. 2 is very difficult to describe analytically [7]. The situation is complicated even further due to nonlinearity of the parasitic capacitances of the semiconductor devices. To accurately predict the behavior of the circuit, a simulation program and precise component models are required.

It was observed that not all reactive elements are necessary for the correct circuit operation, although they will certainly appear in a physical prototype. The absolute minimum requirements are two inductors and two capacitors, either $C_{D S}$ and $C_{I}$, or $C_{R}$ and $C_{I}$. In Fig. 4 the 4-element resonant network seen from the drain terminal is shown, as well as two 3-element simplified networks; note that $L_{I}$ is excluded since it remains the same in all three configurations. With the appropriate components choice, these networks may have exactly the same input impedance at all frequencies, which is the impedance seen by the drain terminals of the MOSFETs. Therefore, in the design process of the proposed interleaved converter, one of the 3-element networks can be used instead, thus reducing the number of unknowns in the design by one. The transformation between the networks in Fig. 4 is given in Table I. Capacitances and inductances are normalized with respect to $C_{I, 1}$ and $L_{R, 1} . n$ and $k$ are the capacitance ratios $C_{D S, 1} / C_{I, 1}$ and $C_{D S} / C_{I, 1}$, respectively. Note that $k$ must always be less than or equal to $n$. This transformation affects the output impedance of the network, which means that the output voltage and current have to be scaled as well in order to obtain identical circuit behavior. For example, if the 3-element network 1 is transformed into the 3-element network 2, output impedance is scaled down by a factor of $(1+n)^{2}$; equivalently, the output current is increased $1+n$ times, and the output voltage is decreased by the same factor.

\section{Interleaved Resonant SEPIC Converter}

In the case of the proposed converter from Fig. 1, it was found that the resonant/switching frequency may be approximately determined from:

$$
f_{S} \approx \frac{1}{2 \pi \sqrt{L_{I}\left(C_{I}+C_{D S, e q)}\right.}}
$$

Capacitance $C_{D S, e q}$ is defined as the equivalent drain to source capacitance:

$$
C_{D S, e q}=C_{O S S}+\frac{C_{1}\left(C_{2}+C_{I S S}\right)}{C_{1}+C_{2}+C_{I S S}}
$$

The exact value of $f_{S}$ also depends on duty cycle of the switch and the diode, which are not easy to predict - especially if nonlinear capacitances of the semiconductor components are taken into account. Nonetheless, equation (1) is a good starting point for the design procedure; reactive elements could be scaled up or down in order to adjust for desired output power or frequency. For example, increase in circuit capacitances $N$ times would result with a converter with $\sqrt{N}$ lower switching frequency and $\sqrt{N}$ higher power output. If capacitances are scaled up and inductances are scaled down by the same factor, 

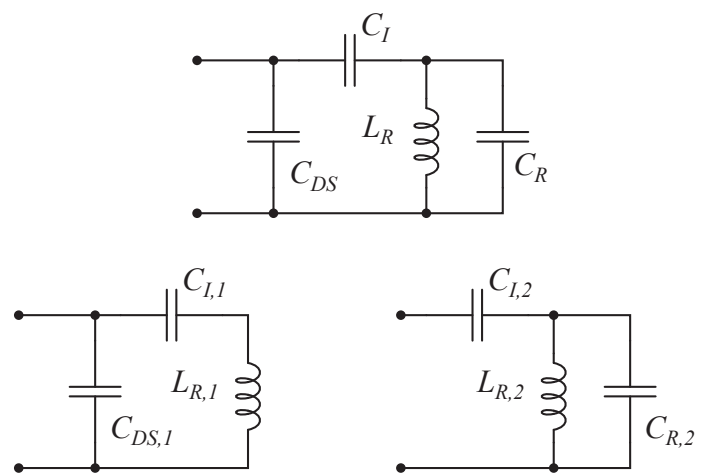

Fig. 4: Resonant network seen by the drain terminal of the MOSFETs, with the exclusion of the input inductor $L_{I}$; original (top) and two alternatives (bottom). The components of these networks may be chosen in such a way that all three circuits have the same input impedance.

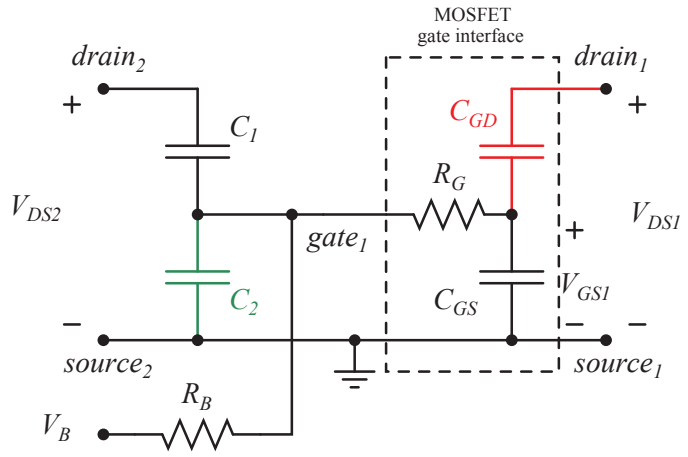

Fig. 5: A part of the interconnection network used with the resonant SEPIC converter to obtain the self-oscillating and interleaved operation. Voltage divider formed by $C_{1}, C_{G S}$, and $C_{2}$ (optional), determines the amplitude of the gate voltage. Note the parasitic feedback from the drain of the same MOSFET. Bias voltage for the gate terminal is provided through resistor $R_{B}$.

Table I: Network transformations.

\begin{tabular}{lcccc}
\hline & $C_{I}$ & $C_{D S}$ & $C_{R}$ & $L_{I}$ \\
\hline original & $(1+n-k) C$ & $k C$ & $(1+n-k)(n-k) C$ & $L /(1+n-k)^{2}$ \\
alternative (1) & $C$ & $n C$ & - & $L$ \\
alternative (2) & $(1+n) C$ & - & $n(1+n) C$ & $L /(1+n)^{2}$ \\
\hline
\end{tabular}

the switching frequency would remain the same and the power output would increase $N$ times.

As it was observed in [7], the waveforms do not deviate significantly with change of voltage transformation ratio $\mathrm{M}$, and this also holds true for the interleaved self-oscillating SEPIC converter. This property is very useful, since it allows easy change of specifications with only minor adjustments to the converter components.

\section{Interconnection Network}

Several gate drive techniques are described in the literature, and in that sense RF DC-DC converters may be separated into two major groups: oscillator-driven [1,2, 5, 7] and self-oscillating converters [4, 6].

The interconnection network from the drain of $S_{2}$ to the gate of $S_{1}$ is shown in Fig. 5. $C_{1}$ and $C_{2}$, together with the gate capacitance of the MOSFET $C_{G S}$, form a capacitive voltage divider, thus generating the signal for driving the other switch.

Gate resistors $R_{B 1}$ and $R_{B 2}$ provide biasing for the MOSFET gates. During the converter startup, $R_{B 1}$ and $R_{B 2}$ form $R-C$ time constants with the gate capacitances of $S_{1}$ and $S_{2}$. In order to reduce the startup time, these time constants should be different, or alternatively, independent bias voltages should be used.

If resistance $R_{G}$ is small compared to impedance of the MOSFET input capacitance $C_{I S S}$ (which is usually the case), then the gate voltage $v_{G S}$ is approximately given as:

$$
v_{G S}(t)=\frac{C_{1}}{C_{1}+C_{2}+C_{G D}+C_{G S}} v_{D S, A C}(t)+\frac{C_{G D}}{C_{1}+C_{2}+C_{G D}+C_{G S}} v_{D S, A C}(t)+V_{B}
$$


The first term in (3) represents feedback from the drain voltage of the opposing MOSFET. The second term is due to parasitic capacitance $C_{G D}$ and is undesirable. The operation point of the switch in the OFFstate might shift into the saturation region and produce significant losses, which also shortens the switch lifetime or, in more severe cases, the switching device may be destroyed. Therefore, it is recommended to use switches with small $C_{G D}$ capacitance in the design, and the feedback network needs to provide enough gain in the primary feedback. This is done by appropriate sizing of $C_{1}$ and $C_{2}$. Additional benefit of having nonzero $C_{2}$ in the circuit which helps suppressing parasitic high frequency oscillations on the gate, which may appear due to stray inductances in the circuit. However, increase of $C_{1}$ and $C_{2}$ is limited by ZVS condition. $C_{I}$ needs to be at least two times larger than $C_{D S, e q}$ in order to achieve ZVS operation.

There are some drawbacks to this technique. In order to obtain circuit symmetry at VHF frequencies, layout needs to be symmetrical and the circuit components should have low tolerances. Since there is a purely capacitive signal path from drain to gate terminals, any parasitic inductance in the circuit might introduce ringing in the gate voltage waveforms. As well as in [6], this self-oscillating scheme shows lower dependency of output power with the bias voltage compared to [9]. Therefore it is reasonable to consider burst-mode control scheme $[1,5,7,10]$ for the proposed converter, which is a subject of further research.

\section{Converter Startup}

Start-up of the circuit is achieved by setting the gate bias voltage slightly above the MOSFET threshold voltage, so the switching devices enter saturation mode. A simplified AC equivalent of the inverter side is shown in Fig. 6. The MOSFETs are modeled as voltage controlled current sources.

The loop formed by the MOSFETs and the capacitive voltage dividers is unstable; hence, amplitudes of the drain (and consequently, gate) voltages increase. This increase is bounded by the fact that the MOSFETs eventually start entering the ohmic region, thus limiting the effective gain in the loop. In the steady state, the MOSFETs do not enter saturation region.

Typical startup gate and drain waveforms are shown in Fig. 7, as a response to a step change in $V_{B}$ at $t=0.2 \mu \mathrm{s}$. Until the oscillations start, the response of the gate voltages is described completely by time constant $\tau_{G}$ defined as

$$
\tau_{G}=R_{B}\left(C_{I S S}+C_{D G}+C_{1}+C_{2}\right)
$$

and ends when the MOSFETs enter saturation. For the circuit in Fig. 6 to be unstable, the following condition has to be satisfied:

$$
g_{m} Q Z_{0}>\frac{1}{x}
$$

where $Q$ is the Q-factor of the resonant tank, $Z_{0}$ is the reactance of the inductors/capacitors at the resonant frequency, $g_{m}$ is the MOSFET transconductance, and $x$ is the scaling factor of the capacitive voltage divider in (3). For a given switching frequency and power level, $g_{m}, Q$, and $Z_{0}$ should be maximized in order to minimize the transient time.

\section{Design Procedure}

A method was proposed in [7] which relies on separate design of the inverter and rectifier stages. However, that procedure has been found unsuitable for the self-oscillating interleaved converter design. As it was discussed in the previous section, it is very difficult to determine the switching frequency a priori due to complexity of circuit operation. More importantly, it is not guaranteed that a converter designed to operate at a certain frequency might not operate at that particular frequency when used in the interleaved 


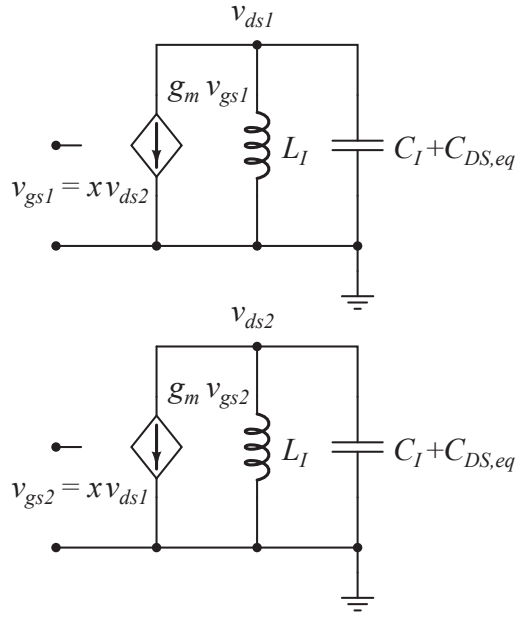

Fig. 6: Simplified AC equivalent circuit of the inverter stages during startup process. The MOSFETs, while in saturation, are modeled as voltage controlled current sources.
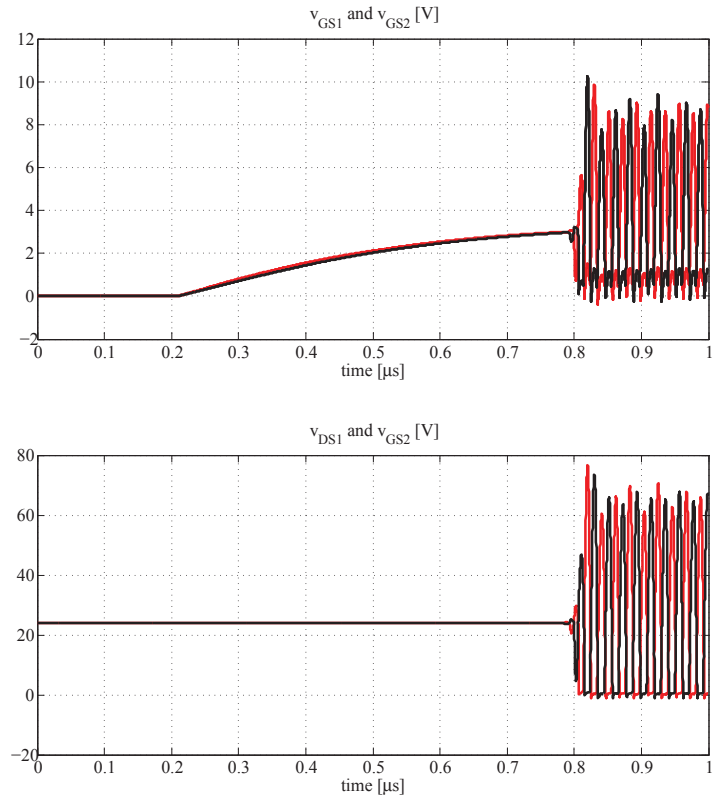

Fig. 7: Gate (top) and drain (bottom) waveforms during the converter startup.

design. Therefore, a direct synthesis method is proposed in this section.

The design procedure starts from specifications for output power $P_{O U T}$, switching frequency $f_{S}$, and input voltage $V_{I N}$. Required component values will be finally determined from the output power $P_{O U T}$ specification by scaling of the reactive components. At this stage of the design process, initial values for $C_{R}$ and $L_{R}$ are $C_{R}=0$ and $L_{R}=1.5 L_{I}$.

Capacitance $C_{1}$ is directly determined from (3):

$$
C_{1}=\left(C_{2}+C_{G S}+C_{G D}\right) \frac{V_{G S, \max }}{V_{D S, \max }-V_{G S, \max }}
$$

where $C_{2}$ may be chosen arbitrarily as long as it does not yield high capacitance value for $C_{1}$. For low voltage applications or when the $C_{G S}$ is large, $C_{2}$ may be omitted. The ratio between the SEPIC "flying" capacitance $C_{I}$ and equivalent drain to source capacitance $C_{D S, e q}$ is a limiting factor for ZVS operation; a good initial value for $C_{I}$ was found to be at least 2-3 $C_{D S, e q}$. The transformation provided in Table I should be applied to include $C_{R}$ in the final design. After $C_{I}$ is selected, input inductance $L_{I}$ is determined from (1):

$$
L_{I}=\frac{1}{\left(2 \pi f_{S}\right)^{2}} \frac{1}{C_{I}+C_{D S, e q}}
$$

Inductor $L_{R}$ is then adjusted as necessary to reach ZVS operation. Impedance scaling is then applied to obtain desired frequency and output power.

The peak drain voltage $V_{D S, \max }$ is expected to be around 3 times higher compared to $V_{I N}$. Therefore, the MOSFET has to be chosen with a voltage rating higher than $V_{D S, \max }$.

Nonlinearities of the parasitic capacitances may influence the final result, and for that reason accurate modeling is beneficial. Nevertheless, even with the simple models of the transistors and diodes reasonable accuracy is obtainable. 


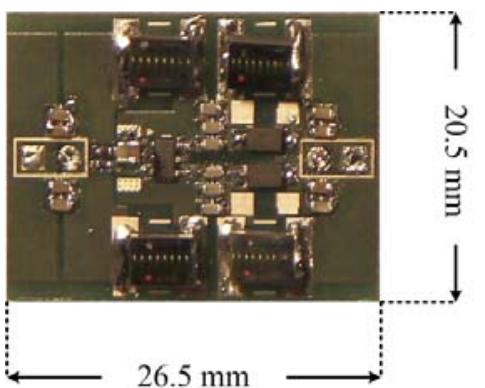

Fig. 8: Experimental DC-DC converter.
Table II: Converter component values.

\begin{tabular}{lr}
\hline Component & Value \\
\hline \hline$L_{I}$ & Coilcraft air-core $82 \mathrm{nH}$ \\
$L_{R}$ & Coilcraft air-core $120 \mathrm{nH}$ \\
$C_{1}$ & C0G $13 \mathrm{pF}$ \\
$C_{2}$ & - \\
$C_{I}$ & $\mathrm{C} 0 \mathrm{G} 80 \mathrm{pF}$ \\
$C_{S, E X T}$ & - \\
$C_{I N}$ & $\mathrm{C} 0 \mathrm{G} 8 \mathrm{uF}$ \\
$C_{O U T}$ & $\mathrm{COG} 8 \mathrm{uF}$ \\
$\mathrm{S}$ & Fairchild FDC8602 \\
$\mathrm{D}$ & NXP PMEG6010 \\
\hline
\end{tabular}

\section{Experimental Results}

Experimental setup was built to verify the proposed design procedure (see Fig. 8). The DC-DC converter specifications are as follows:

- input voltage $V_{I N}=24 \mathrm{~V}$

- output voltage $V_{O U T}=11 \mathrm{~V}$

- output current $I_{O U T}=1$ A

- switching frequency $f_{S}=50 \mathrm{MHz}$

A complete list of components used is presented in Table II.

The MOSFET device to be used in the design needs to be able to withstand at least 3 times the input voltage. Therefore, $100 \mathrm{~V}$ rated devices were considered, and FDC8602 dual MOSFET from Fairchild Semiconductor was chosen since it offered a good balance between $R_{D S, \text { on }}$ and parasitic capacitances $C_{I S S}$ and $C_{\text {OSS }}$. On the other hand, the downsides are the presence of the stray inductance and high thermal resistance of the package. Experimental voltage waveforms of the designed converter are provided in Fig. 9. Note the high-frequency ringing due to finitely small inductances of the component packages and PCB traces.

The circuit shows little tolerance to non-symmetry, therefore direct measurements with a $9.5 \mathrm{pF}$ oscilloscope probe would disturb the operation of the converter, especially of $v_{D}$ and $v_{R}$ nodes. The measurements of these nodes were obtained through $2.7 \mathrm{pF}$ capacitance instead (see Fig. 10), which gives the scaling factor of 0.22 and removes the DC component from the waveform. With input voltage of $24 \mathrm{~V}$, expected peak voltage across the MOSFETs should be around $72 \mathrm{~V}$. In Fig. 9a, peak-to-peak $v_{D S}$ voltages are $15 \mathrm{~V}$. This gives $V_{D S, \max }$ of $68 \mathrm{~V}$ which matches well with expectations.

$v_{G S}$ was designed to have $10 \mathrm{~V}$ peak-to-peak value. In Fig. $9 \mathrm{~b}$ the direct measurement is provided, and it can be observed that the amplitude is close to $8 \mathrm{~V}$, indicating the influence of the probe capacitance. Measured switching frequency was $48.7 \mathrm{MHz}$ when the probe was connected to the board via $2.7 \mathrm{pF}$ capacitor. The frequency is $5 \%$ off the target value, which is accredited mainly to the simplicity of the model and equation (1). 


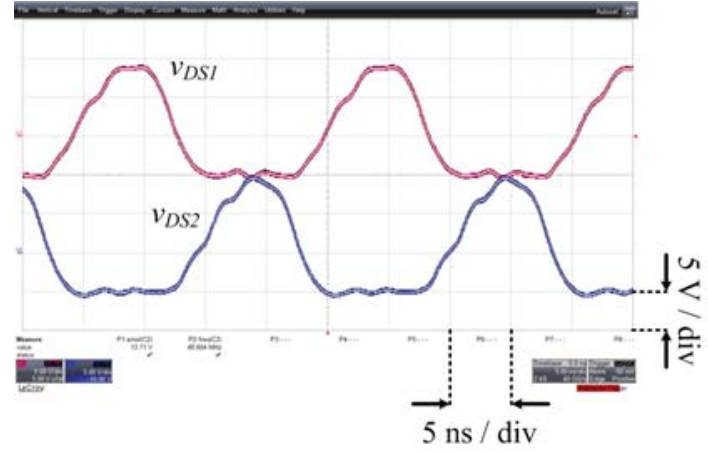

(a) Drain voltages $v_{D S 1}(t)$ and $v_{D S 2}(t)$

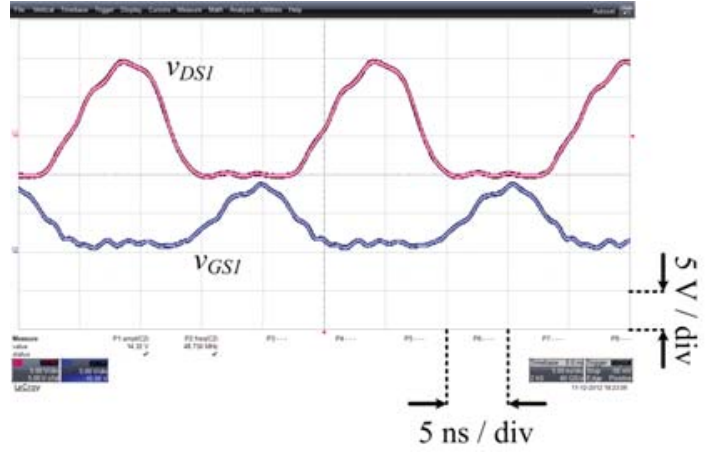

(b) Drain and gate voltages $v_{D S 1}(t)$ and $v_{G S 1}(t)$

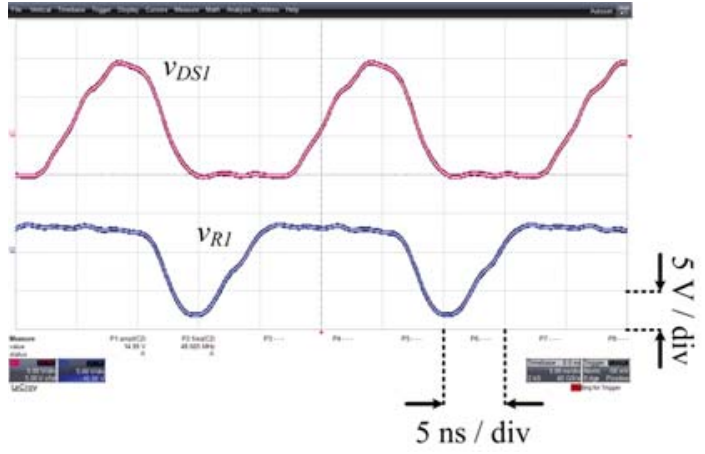

(c) Drain and rectifier voltages $v_{D S 1}(t)$ and $v_{R 1}(t)$

Fig. 9: Experimental waveforms of the prototype converter.
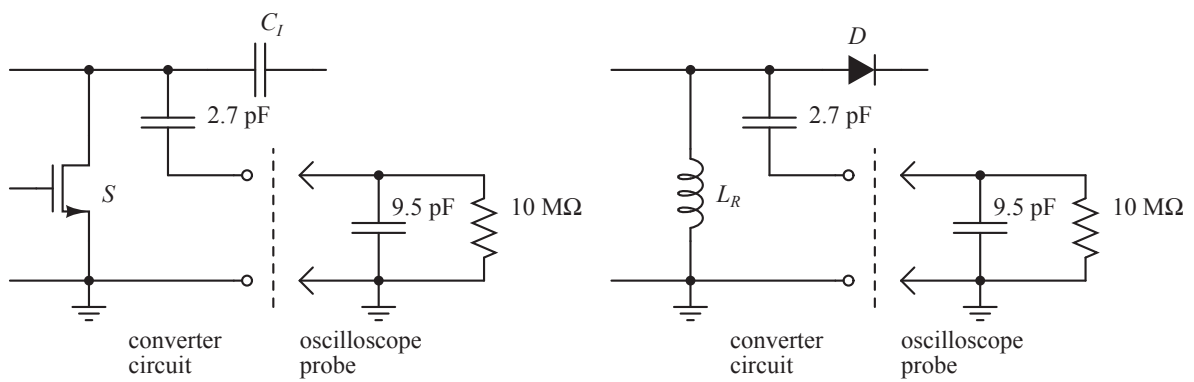

Fig. 10: Measurement setup for drain (left) and rectifier (right) voltages.

\section{Conclusion and Future Work}

This paper presents a gate driving scheme which provides self-oscillating and interleaved operation of two resonant SEPIC converters. The only components of the gate drive which do not belong to the converter topology are two capacitors and two biasing resistors. These components are typically small in size, which makes this gate drive method very component and space efficient. A design procedure for the interleaved SEPIC converter has been proposed, and experimental results have verified its effectiveness. A 50-MHz, $11 \mathrm{~W}$ converter has been designed and implemented, using only low-cost diodes and VDMOS switches. The peak achieved efficiency was $87 \%$.

The circuit layout needs to be as symmetrical as possible in order to achieve optimal operation. Output power is not significantly influenced by variation of the gate bias voltage. As part of the ongoing re- 
search, implementation of the burst-mode control with this gate driving technique will be investigated, for which this gate drive technique shows promising results.

As this paper has demonstrated, it is possible to obtain acceptable efficiency, small converter size, and low cost in VHF power conversion.

\section{References}

[1] R. C. N. Pilawa-Podgurski, A. D. Sagneri, J. M. Rivas, D. I. Anderson, and D. J. Perreault, "Very-HighFrequency Resonant Boost Converters," IEEE Trans. Power Electron., vol. 24, no. 6, pp. 1654-1665, Jun. 2009.

[2] J. M. Rivas, D. Jackson, O. Leitermann, A. D. Sagneri, Y. Han, and D. J. Perreault, "Design Consideration for Very High Frequency DC-DC Converters," in IEEE Power Electronics Specialists Conference (PESC), Ottawa, Canada, Jun. 2006, pp. 1-11.

[3] M. Alimadadi, S. Sheikhaei, G. Lemieux, S. Mirabbasi, W. G. Dunford, and P. R. Palmer, "A Fully Integrated $660 \mathrm{MHz}$ Low-Swing Energy-Recycling DC-DC Converter," IEEE Trans. Power Electron., vol. 24, no. 6, pp. 1475-1485, Jun. 2009.

[4] T. Andersen, S. Christensen, A. Knott, and M. A. E. Andersen, "A VHF Class E DC-DC Converter with Self-Oscillating Gate Driver," in Proc. Applied Power Electronics Conference and Exposition (APEC), San Francisco, California, USA, Sep. 2011, pp. 885-891.

[5] J. M. Rivas, O. Leitermann, Y. Han, and D. J. Perreault, “A Very High Frequency DC-DC Converter Based on a Class $\Phi_{2}$ Resonant Inverter," IEEE Trans. Power Electron., vol. 26, no. 10, pp. 2980-2992, Oct. 2011.

[6] M. Kovacevic, A. Knott, and M. A. E. Andersen, "Interleaved Self-Oscillating Class E Derived Resonant DC/DC Converters," in Proc. International Conference on Electrical and Computer Systems (ICECS2012), Ottawa, Canada, Aug. 2012, pp. (192-)1-8.

[7] J. Hu, A. D. Sagneri, J. M. Rivas, Y. Han, S. M. Davis, and D. J. Perreault, "High Frequency Resonant SEPIC Converter with Wide Input and Output Voltage Ranges," IEEE Trans. Power Electron., vol. 27, no. 1, pp. 189-200, Jan. 2012.

[8] M. Kazimierczuk and J. Jozwik, "Resonant DC/DC converter with class-E inverter and class-E rectifier," IEEE Trans. Power Electron., vol. 36, no. 4, pp. 468-478, Nov. 1989.

[9] P. Shamsi and B. Fahimi, "Design and Development of VHF Resonant DC-DC Boost Converters," IEEE Trans. Power Electron., vol. 27, no. 8, pp. 3725-3733, Jun. 2012.

[10] I. J. R. Warren, K. A. Rosowski, and D. J. Perreault, “Transistor Selection and Design of a VHF DC-DC Power Converter," IEEE Trans. Power Electron., vol. 23, no. 1, pp. 27-37, Jan. 2008. 